\title{
RESEARCH ON DYNAMIC CHARACTERISTICS OF 5-DOF SERVICE ROBOT MANIPULATOR
}

\author{
Li Yang ${ }^{1}$, Cheng Weiming ${ }^{2}$, Gong Chengwen Bian Jiajia ${ }^{3}$ \\ ${ }^{1}$ School of Mechanical Engineering, Shanghai University of Engineering Science, Shanghai 201600 \\ ${ }^{2}$ School of Mechanical Engineering, Shanghai University of Engineering Science, Shanghai 201600 \\ ${ }^{3}$ School of Mechanical Engineering, Shanghai University of Engineering Science, Shanghai 201600
}

\begin{abstract}
In view of the less application scene of the family service robot in real life, the design scheme of the 5-DOF manipulator is determined and its main dynamic characteristics are studied in the actual life of the multi-freedom manipulator. The joint structure of the 5-DOF manipulator is designed. The distribution of joint freedom and the selection of joint motor and reducer are introduced, and the connection mode of motor and reducer is introduced. The dynamic equation based on Lagrange is designed, and the main parameters affecting the dynamic characteristics are deduced. A simulation based on speed and acceleration is designed to verify the accuracy of the dynamics. The application results show that the whole design scheme is reliable and can achieve the designed target.
\end{abstract}

Keywords: - Service Robot; Dynamics Analysis; Lagrange; Simulation Analysis

\section{INTRODUCTION}

With the development of the times and technological progress, in order to solve the problem of population and the rapid growth of labor costs, robot technology is emerging one after another. In the production of highly intelligent modern factories, the application of industrial robots can be seen everywhere, but the service robots for home applications have not yet popularized[1]. Compared with industrial robots, service robots attach more importance to human-machine interaction experience, and interaction between users and robots is more frequent. As an important part of service robot, mechanical design and dynamic analysis can promote the development and progress of service robots.

Zhang Rui[2]2905 gives a detailed introduction to the overall design of the service robot, focusing on the design of the legs and the hardware of the service robot, and the rational distribution of the overall degree of freedom of the robot. Feng Yuqian and other people[3], based on the dynamics analysis of the manipulator using the Lagrange equation for the 7 degree of freedom manipulator, the function curves of the dynamic moments of each joint are obtained, the rationality of the model is verified, and the corresponding numerical analysis is carried out with the Matlab software to verify the feasibility of the design of the whole method. People such as Guo Dabao[4], based on the Newton Euler dynamic equation, proposed a lightweight design method of a series six joint manipulator with an old man's service robot. The static and dynamic simulation analysis of the manipulator was carried out by CAE software, and the suitable material was selected to optimize the structure. Wu Liangkai and other people[5] use the
Lagrange method to establish the dynamics model of the mechanical arm, and use the SolidWorks to build the threedimensional space assisted mechanical arm. The dynamic analysis is carried out in the ADAMS, which provides the basis for the structure design and the optimal control.

This paper mainly introduces the design requirements of the robot arm of the service robot, and introduces the distribution of the degree of freedom of the manipulator and the selection of the joint motor. On the basis of completing the design of manipulator mechanism, a dynamic model is built and analyzed in the simulation software. The simulation software is used to set the different initial conditions of the manipulator, and the correlation analysis of speed and acceleration is carried out respectively. The accuracy of the dynamic model is verified, which proves the integrity and reliability of the scheme.

\section{THE STRUCTURE DESIGN}

The most important applications of service robots are families, such as serving tea for the elderly, or sending water. Taking into account the overall appearance and working conditions of the robot. At the beginning of the structural design, combined with actual demand and market feedback. The overall structure and joint size of the manipulator are designed according to the human arm. This provides the necessary parameters for subsequent parametric design and dynamic analysis. The whole arm is divided into three parts: shoulder, elbow and wrist. Specific size and its weight, as shown by Table 1: 
Table 1: Manipulator size and weight

\begin{tabular}{|l|l|l|l|}
\hline & shoulder & elbow & wrist \\
\hline size $(\mathrm{mm})$ & 150 & 250 & 300 \\
\hline weight $(\mathrm{kg})$ & 2 & 2.5 & 1.5 \\
\hline
\end{tabular}

The basic parameters of the structural design are: the overall quality is $6 \mathrm{~kg}$, the total length is $700 \mathrm{~mm}$, and the terminal load is $1 \mathrm{~kg}$. According to the speed of human arm movement, the maximum angular velocity of the joint rotation of the manipulator is set to $2 \mathrm{rad} / \mathrm{s}$, and the maximum line speed of the end point is $2 \mathrm{~m} / \mathrm{s}$.

\subsection{The Freedom of Joint}

The key step in the design of manipulator structure is the distribution of the degree of freedom of the manipulator, and reasonable allocation can optimize the overall structure. For the design of the robot arm of a service robot, the more freedom the degree of freedom is, the more flexible the manipulator is, but the corresponding is that the overall structure becomes very complex, which is not conducive to the design and manufacture of the manipulator. According to the degree of freedom distribution scheme of Reference[2], combined with the needs of practical applications, the joints were classified into shoulder joint, elbow joint and wrist joint. There are 2 degrees of freedom in the shoulder joint, 1 degrees of freedom in the elbow joint, and 2 degrees of freedom in the wrist joint. The 3 degrees of freedom of the shoulder joint and elbow joint determine the space position of the manipulator, and the two degrees of freedom of the wrist joint determine the end posture of the manipulator.

\subsection{Mechanical Structure}

The structure of the manipulator, as shown in Figure 1

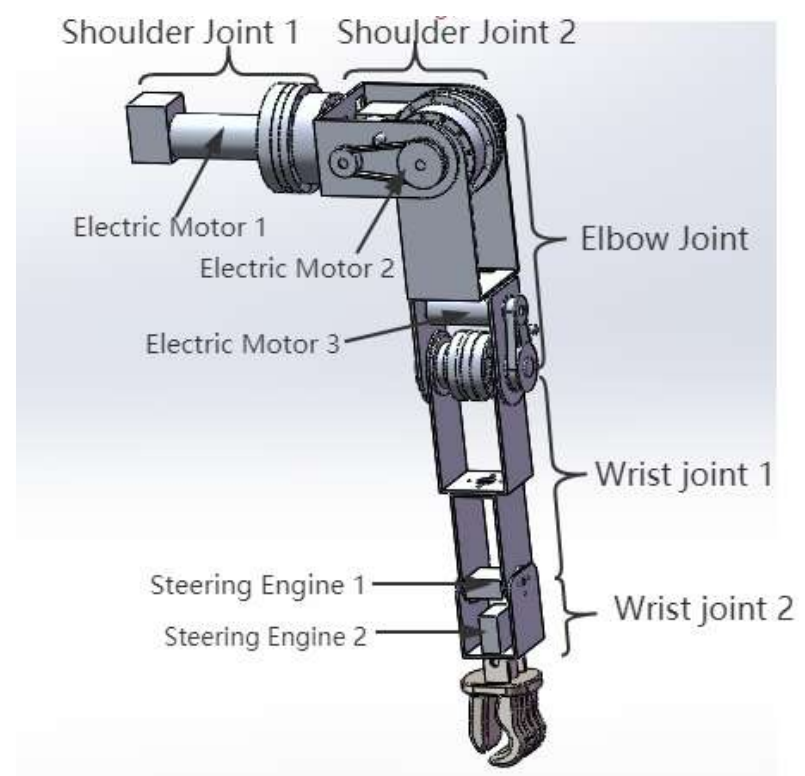

Fig 1: The structure of the manipulator

In the structure of the manipulator, the most important thing is the choice of the motor and the reducer. Considering the motion precision of the whole manipulator, the DC servo motor is selected in the motor 1,2, and 3 of the shoulder joint and elbow joint. The corresponding reducer chooses the harmonic reducer, which can meet the demand of torque and power and improve the motion precision. For the wrist, the position precision is not high, the main purpose is to adjust the end attitude of the manipulator. Under the condition of the joint size, the steering gear can be selected to reduce the joint size effectively. The synchronous belt is connected to the joint of the motor 2, 3 and the retarder of the shoulder joint and the elbow joint. It can effectively reduce the size of the joint, which is beneficial to the dexterity and beauty of the structure.

\section{DYNAMIC ANALYSIS OF MANIPULATOR}

Lagrange's dynamic equation is an equation based on energy conservation, which is applicable to the dynamic analysis of multiple links with constraints[3]. As shown in Figure 1, theta1- theta5 is the rotation angle corresponding to the arm shoulder joint 1 , the shoulder joint 2 , the elbow joint, the wrist joint 1 and the wrist joint $2, \mathrm{~m} 1-\mathrm{m} 5$ is the corresponding joint quality, the $\mathrm{m} 0$ is the load weight, and the T1-T5 is the torque of the joint output torque (the torque after the motor through the deceleration mechanism). Thus, according to the Lagrange equation, it can be obtained:

$$
\begin{aligned}
& L=K-P= \\
& \frac{1}{2} \sum_{i=1}^{n} \sum_{j=1}^{i} \sum_{k=1}^{i} \operatorname{Trace}\left(U_{i j} J_{i} U_{i k}^{T}\right) \dot{\theta}_{j} \dot{\theta}_{k}+\frac{1}{2} \sum_{i=1}^{n} I_{i} \dot{\theta}_{i}^{2}-\sum_{i=1}^{n}\left[-m_{i} g^{T} \cdot\left(T_{i} \bar{r}_{i}\right)\right]
\end{aligned}
$$


Formula(1), $\mathrm{L}$ is the Lagrange function, $\mathrm{K}$ is the kinetic energy of the system, $\mathrm{P}$ is the system potential energy, is the length of the arm corresponding to the joint.

$$
\begin{aligned}
& \mathrm{T}_{i}=\frac{\partial}{\partial t}\left(\frac{\partial L}{\partial \dot{\theta}}\right)-\frac{\partial L}{\partial \dot{\theta}} \\
& =H(\theta) \ddot{\theta}+C(\theta, \dot{\theta}) \dot{\theta}+G(\theta) \\
& =\sum_{j=1}^{n} \sum_{p=\max (i, j)}^{5} \operatorname{Trace}\left(U_{\mathrm{P} i} J_{p} U_{p i}^{T}\right) \ddot{\theta}_{j}+I_{i} \ddot{\theta}_{i}+\sum_{j=1}^{n} \sum_{k=1}^{n} \sum_{p=\max (i, j)}^{5} \operatorname{Trace}\left(U_{P i k} J_{p} U_{p i}^{T}\right) \dot{\theta}_{j} \dot{\theta}_{k}+\sum_{p=i}^{5}\left(-m_{p} g^{T} U_{p i} \bar{r}_{p}\right)
\end{aligned}
$$

In the formula (2), it mainly includes four items, the first two are the acceleration of the motion of the manipulator, which represents the influence of the inertia force and the inertia of the mechanism body on the torque of the joint. The third item in the formula is the influence of the force and centripetal force, and the last one is the influence of the joint gravity on the joints. To sum up, it is clear that, for the dynamic analysis of a joint, it affects the rotational speed and acceleration of the joint, the gravity of the joint itself and the size of the joint.

\section{DYNAMICS SIMULATION OF}

\section{MANIPULATOR}

After determining the overall structure and the corresponding parameters of the manipulator, the model is introduced into the professional simulation software for the corresponding parameters design, environment construction and simulation analysis, to verify the feasibility of the overall design.

\subsection{Determination of Environmental Parameters}

The steps to build a manipulator simulation model:

(1) Setting up working environment: mainly including workspace setting, unit setting and coordinate system establishment.

(2) Add material properties to the model: material aluminum alloy, and add the rated load at the end of the hand grasping part.

(3) Add constraint to the model, including 1 fixed pairs (big arm and ground), 5 rotating pairs, such as Figure 2.

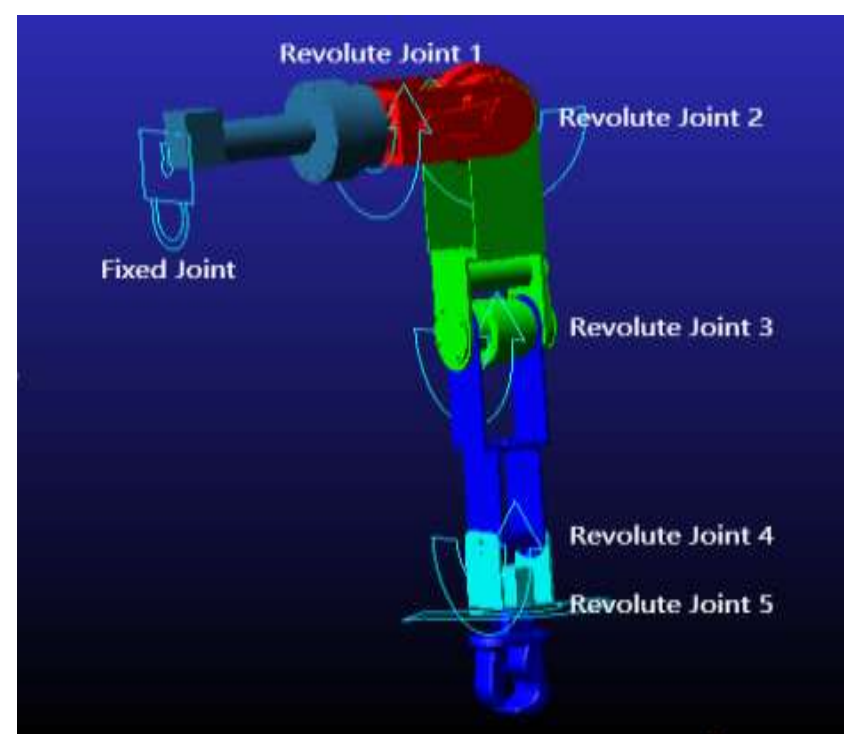

Fig 2: Manipulator joint constraint

\subsection{Simulation Analysis}

After the simulation environment of the manipulator is built, it is necessary to add the driving function to the joints, so as to carry out the dynamics simulation. As shown in Figure 2, the direction of gravity is downward along the manipulator and $1 \mathrm{~kg}$ at the end of the manipulator. The following analysis is based on the velocity of the end point centroid and the rotation pair 1 corresponding to the shoulder motor 1 .

First, in the normal motion of the manipulator, the time of joint acceleration is very short. Therefore, the initial angular velocity of the joint is the maximum $(2 \mathrm{rad} / \mathrm{s})$, and the acceleration is 0 , as shown by Figure 3a. The velocity of the load center varies from $0.4 \mathrm{~m} / \mathrm{s}-2.6 \mathrm{~m} / \mathrm{s}$ to an inverted bell shape. It can be clearly seen from the diagram that the point at which the terminal velocity exceeds the maximum velocity is mainly distributed between $0-0.4 \mathrm{~s}$ and $2.63 \mathrm{~s}$. In combination with Figure 2, several joints at the end of the motion of the manipulator are basically at the same level, the superposition of the speed, which leads to the speed of the end point exceeding $2 \mathrm{~m} / \mathrm{s}$. But in the actual operating environment, the joints of the manipulator generally do not 
move at the same time at the same time at the same time, and in this case, it provides a reference for the design of the control scheme of the subsequent manipulator. For the torque of the rotating pair 1 corresponding to the motor 1 , it is worth the range of $0-4 \mathrm{~N} . \mathrm{m}$, showing a wave shape, for example, at the initial $0-0.7 \mathrm{~s}$, the torque begins to increase, and then begins to fall after the peak, so in the subsequent movement. The reason is that this posture is related to the movement of the manipulator.

Secondly, the angular velocity of the rotation pair 1 is unchanged, and the angular velocity of the other four pairs of rotation pairs is 0 , as shown in Figure $3 \mathrm{~b}$. In this picture, due to the elimination of the influence of several other joint motions on the end load centroid point, the velocity is moving at a uniform speed, while the corresponding torque changes regularly, and the torque range is between $0-11 \mathrm{~N} . \mathrm{m}$, compared with Figure $3 \mathrm{a}$, it can be seen that in the process of mechanical arm movement, the interaction between different joints is very large, and the torque changes significantly. The reason is that the rotation of other joints changes the torque arm length of the corresponding joint. This provides a reliable reference for the improvement of the following mechanical arm structure and the selection of the related motor. Compared with the manual calculation, it can save a lot of time cost.

Finally, based on the motion consideration of the actual manipulator, the driving function is added to the rotary pair 1.

\section{SETP5(time, 0,0rad,0.5,2rad)$$
+\operatorname{SETP5}(\text { time, 0.5,0rad }, 2.5,0 \mathrm{rad})+\operatorname{SETP} 5(\text { time }, 2.5,0 \mathrm{rad}, 3.0,-2 \mathrm{rad})
$$

It represents the 5 order fitting function. The function equation is divided into 3 parts, the initial acceleration motion, the uniform motion in the middle and the deceleration motion at the end. The simulation structure is shown in Figure 3c. In the initial acceleration movement, the joint torque increases and reaches a node. In subsequent uniform motion, the torque increases first and then decreases to 0 . Similar to the case of Figure $3 \mathrm{~b}$, the torque of the manipulator will turn to 0 when it rotates, then the torque will gradually increase, and it will be reduced to 0 at the time it reaches. The variation range of joint torque is basically the same as that of Figure $3 b$, only in the initial state of the motion of the manipulator, it is mainly caused by the acceleration of the joint velocity. This further validates the formula (2), which proves the accuracy of the dynamic model.

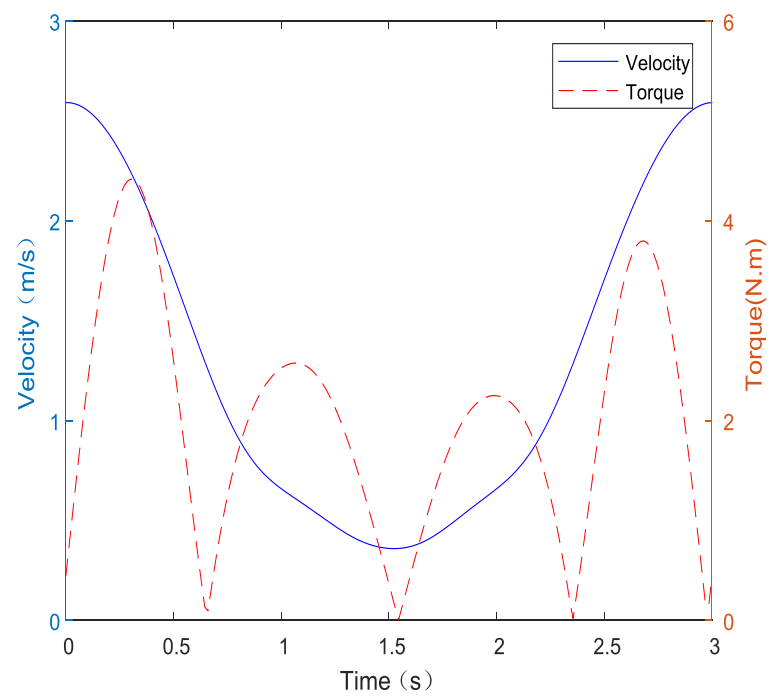

a. The five revolving pairs are the maximum speed

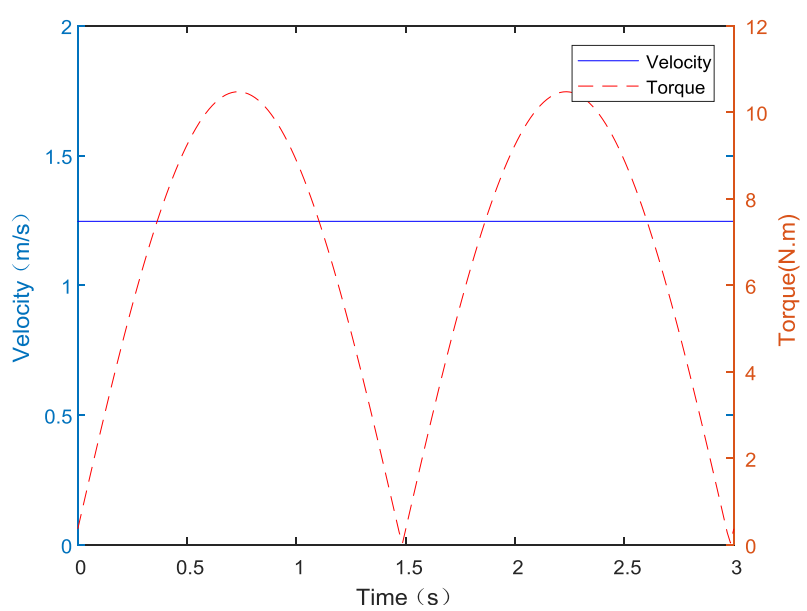

b. The first rotation pair is the maximum speed

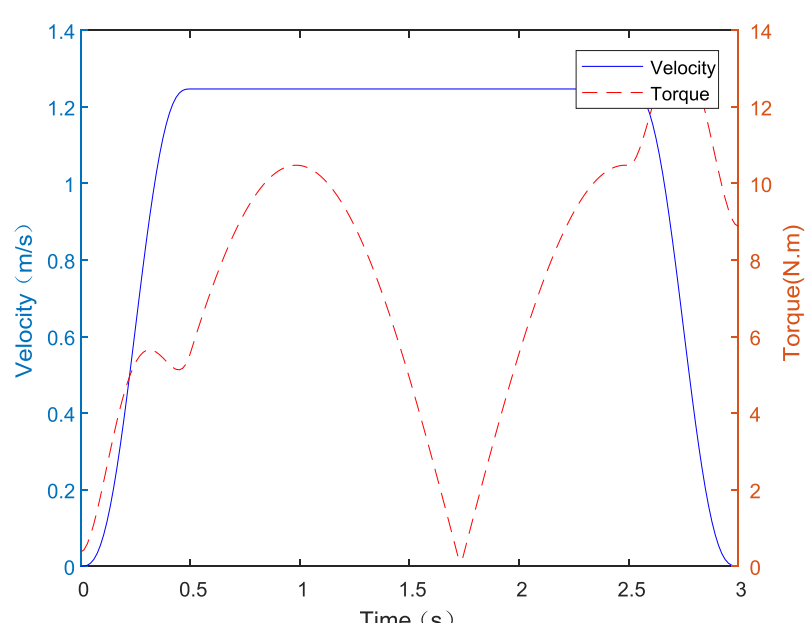

c. The acceleration of the first rotating pair is not 0

Fig 3: Relationship between the load center velocity and the torque of the first rotate pair at the manipulator 


\section{CONCLUSION}

The structure of the five freedom manipulator is designed, the distribution of the joint freedom is determined, the type of the motor and the reducer and the connection mode are given, which provides a feasible scheme for the design of the structure of the manipulator.

Using the Lagrange equation, the influence of velocity and acceleration on the joint torque is analyzed under the condition of mechanical structure and terminal load. The reliability of the results is verified by simulation analysis. It provides a more efficient and reliable method for motor selection.

\section{REFERENCES}

[1] Ji Pengcheng, Shen Huiping. Current situation and development trend of service robot [J]. Journal of Changzhou University, 2010, 22 (02): 73-78.

[2] R. Zhang, "Design and application of innovation platform of full-size humanoid robots," 2017 Chinese Automation Congress (CAC), Jinan, 2017, pp.29042907.

[3] Feng Yuqian, Gao Jinhong, Wang Dianjun, Du Longbo. Dynamic analysis and simulation of seven degrees of freedom robot based on ADAMS [J]. Journal of Beijing Institute of Petrochemical Technology, 2013,21 (02): 39-44.

[4] Guo Dabao, Mei Tao, Luo Minzhou, Feng Baolin, Zhao Jianghai. Dynamic analysis and lightweight design of robot arm for elderly service robot $[\mathrm{J}]$. China Mechanical Engineering, 2012,23 (10): 11461150 .

[5] Wu Liangkai, Wang Tao, Wang Chunli, Wang Zhou, Xia Guohui. Dynamics modeling and simulation analysis of three dimensional space manipulator $[\mathrm{J}]$. Mechanical Engineer, 2017 (01): 15-17.

[6] Zhu Yong, Zhou Siyue. Dynamic modeling of robot arm system [J]. Mechanical \&Electronic, 2008 (03): 60-63.

[7] Liu Mingzhi, Liu Chunxia. Research on Dynamics Modeling and control of flexible manipulators [J]. Advances in Mechanics, 2001 (01): 1-8.

[8] Xuping Zhang,Rasmus Sørensen,Mathias Rahbek Iversen,Haijie Li. Computationally efficient dynamic modeling of robot manipulators with multiple flexible-links using acceleration-based discrete time transfer matrix method[J]. Robotics and Computer Integrated Manufacturing,2018,49.

[9] Li Yaowen, Li Qunming, Duan Xiaogang. Dynamic modeling and fuzzy sliding mode control of five degree of freedom manipulator [J]. Modern Manufacturing Engineering, 2016 (06): 34-39.

\section{BIOGRAPHY}

Li Yang, male, han, graduate student of Shanghai university of engineering and technology, research direction: Design and control of robot manipulator.
Address: 1000 21\#3006, Wenhui Road, Songjiang District, Shanghai

Zip code : 201600

Contact number : 18017066105

Mailbox : 18017066105@163.com 